\title{
Pneumocystis carinii pneumonitis: a serological study
}

\author{
VALERIE SHEPHERD ${ }^{1}$, BERYL JAMESON ${ }^{2}$, AND G. K. KNOWLES 3 \\ From the ${ }^{1}$ Institute of Cancer Research and the ${ }^{2}$ Royal Marsden Hospital, Sutton, Surrey; and the \\ ${ }^{3}$ Professorial Unit, Cardiothoracic Institute, London SW3 6HP, UK
}

SUMMARY Sera from 23 proven or clinically suspected cases of Pneumocystis carinii pneumonitis (PCP) in immunosuppressed patients predominantly with acute lymphoblastic leukaemia, and from 91 normal people, were examined for antibody to $P$. carinii by indirect immunofluorescence. Low levels of antibody were found in 51 of 91 normal people and elevated or rising titres of antibody in 18 out of 21 cases from whom paired or serial convalescent sera could be obtained.

Pneumocystis carinii pneumonitis (PCP), a complication of immunosuppression, although commonly reported in the United States of America (Singer et al., 1975; reviewed by Hughes, 1975) has until recently been regarded as a rarity in the UK (Lipson et al., 1977). The occurrence, beginning in November 1974 , of 13 proven and 19 clinically suspected cases during a period of $\mathbf{3 4}$ months at the Royal Marsden Hospital, Surrey, where only three cases had been identified in the previous 10 years, prompted a search for a presumed source of infection among the patients' contacts. Examination of sera from healthy family members and hospital staff revealed the presence of apparently specific antibody in titres up to $1: 32$ in a high proportion of them. This finding was repeated in a further group of healthy people with no known contact with PCP.

Serology could not, therefore, be used for epidemiological studies, but its value in diagnosis was investigated by the subsequent examination of serial sera from cases of PCP during the acute and convalescent stages of the infection.

\section{Patients and methods}

Sera from 23 patients were studied. In 10 cases the diagnosis of PCP was confirmed by the demonstration of pneumocysts in lung tissue, obtained by needle aspiration in eight and by biopsy in two. The remaining 13 were diagnosed on clinical and chest $x$-ray evidence, together with response to either pentamidine isethionate or high-dose co-trimoxazole (Hughes et al., 1975; Lau and Young, 1976). Two

Received for publication 30 November 1978 patients died soon after the onset of infection and only admission sera were available. Admission plus convalescent sera (from two to eight samples per patient) were obtained from 18 patients during a prospective study carried out at the Royal Marsden Hospital; stored sera from five past cases were contributed to the investigation by the London and Brompton Hospitals.

In addition, sera were examined from 91 healthy adults and children, 31 of whom were either family or nursing contacts of the patients and 60 who had no known contact with any case of PCP. A small group of eight control patients, matched with eight proven cases of PCP for age, sex, underlying diagnosis, and duration of treatment, were also included to determine whether their antibody findings resembled those of the known cases or of the normal group.

Tables 1 and 2 summarise the details of the patients in the confirmed and suspected groups.

SEROLOGICAL STUDIES

Sera stored at $-20^{\circ} \mathrm{C}$ were examined for antibody

Table 1 Confirmed cases of PCP

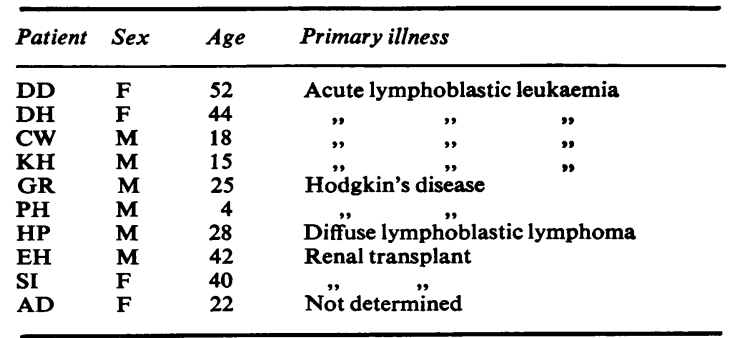


Table 2 Suspected PCP

\begin{tabular}{|c|c|c|c|c|c|}
\hline Patient & $\operatorname{Sex}$ & Age & \multicolumn{3}{|c|}{ Primary illness } \\
\hline JK & $\mathbf{M}$ & 11 & \multicolumn{3}{|c|}{ Glioma } \\
\hline IK & $\mathbf{F}$ & 50 & \multicolumn{3}{|c|}{ Idiopathic thrombocytopenic purpura } \\
\hline EC & $\mathbf{F}$ & 57 & \multicolumn{3}{|c|}{ Acute lymphoblastic leukaemia } \\
\hline GF & $\mathbf{M}$ & 34 & , &, & ", \\
\hline JD & $\mathbf{F}$ & 20 & , & $"$, & ", \\
\hline CS & $\mathbf{F}$ & 18 & , & $"$ & ", \\
\hline VM & $\mathbf{F}$ & 17 & " & $"$ & ", \\
\hline SR & $\mathbf{M}$ & 14 & , & , & $"$ \\
\hline $\mathbf{A P}$ & $\mathbf{F}$ & 14 & ", & ", & ", \\
\hline SW & $\mathbf{M}$ & 12 & ," & ", & " \\
\hline KF & $\mathbf{M}$ & 10 & , & ", & ", \\
\hline $\mathbf{A N}$ & $\mathbf{F}$ & 5 & , & $"$ & ", \\
\hline SL & $\mathbf{F}$ & 3 & ", & ", & ", \\
\hline
\end{tabular}

to $P$. carinii by Coon's indirect immunofluorescence technique using, as the substrate, paraffin sections of human lung heavily infested with pneumocysts kindly provided by $\operatorname{Dr} A$. Nowoslawski of the National Institute of Hygiene in Poland. The tissue had been fixed in Bouin's fluid to destroy existing immunoglobulin in the alveolar exudate and to preserve polysaccharide antigen. The sections were deparaffinised, rehydrated, and washed for 30 minutes in phosphate-buffered saline ( $\mathrm{pH} \mathrm{7 \cdot 4)}$ before application of the staining reagents. All sera were initially screened at a 1:8 dilution with fluoresceinlabelled anti-human immunoglobulin (Wellcome Reagents). Positive sera were subsequently titrated for IgG and IgM antibodies in doubling dilutions from 1:8 upwards, using class-specific antisera. Positive and negative controls were included in each batch. A positive reading was recorded when the entire content of the alveolar spaces (which include all stages of the parasite's development) was brightly stained (see Fig. 1).

Good agreement was obtained in a preliminary assessment in which two observers (VS and BJ) allocated slides to one of five categories (negative, $\pm,+,++,+++)$ according to the brightness of the fluorescence. Titres were reported as the highest dilution giving a one-plus reading.

\section{IMMUNOGLOBULIN ESTIMATIONS}

Levels for the three major Ig classes-IgG, IgM, and $\operatorname{Ig} \mathrm{A}$-were measured in patients' admission sera by the Ouchterlony double diffusion technique using Hoechst partigen plates. The purpose was to see if low immunoglobulins might be associated with failure to produce measurable $P$. carinii antibody.

\section{Results}

The results are summarised in Table 3. The highest IgG titre recorded in the two healthy control groups was $1: 32$. The proportion of individuals with evidence of antibody was the same in both patients' contacts and in those with no known contact. IgM titres did not exceed $1: 8$.

The titres shown in Table 3 for proved or suspected cases of PCP are the highest IgG antibody levels measured at any stage of the illness. Sera were obtained from patients at variable intervals, so the highest level recorded for a given case may not necessarily be the maximum reached (Fig 2).

Ten patients attained antibody levels greater than the highest for the two control groups. Seven others

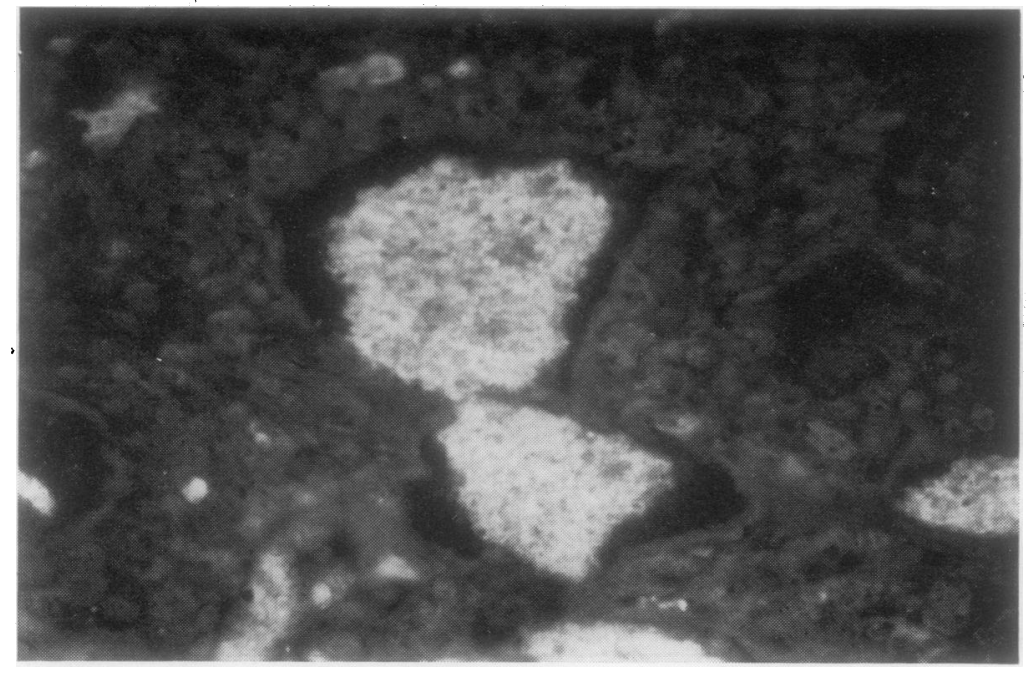

Fig. 1 Section of human lung showing alveoli packed with pneumocysts which are stained with fluorescent antihuman IgG after incubation with a 1/32 dilution of serum from a proven case of PCP. Magnification $\times 1200$. 
Table 3 Highest titres of IgG antibody

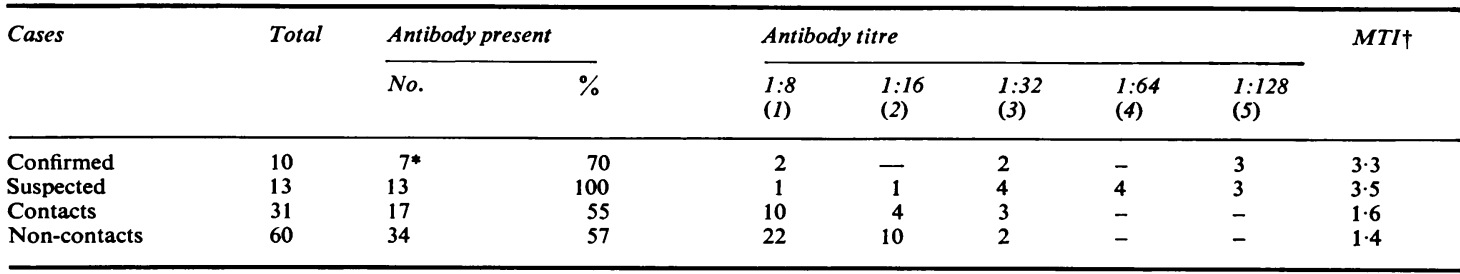

* Two of the 3 negative confirmed cases died when only admission sera had been collected.

†The mean titre index (MTI) is obtained by applying an arbitrary score of 1-5 to each antibody dilution (see above) and calculating the arithmetic mean of the total scored in each group.

had highest levels within the upper limit of the normal range but five of these showed a four-fold rise. Three converted from negative to $1: 8$; one proven case remained negative. In the patients whose antibody titre reached $1: 128$, this level was measurable at 10-14 days after the onset of the acute phase.

Only three of eight patients matched for age, sex, and disease but without respiratory illness were found to have antibody. All were within the range recorded in the healthy control groups.

\section{Discussion}

No completely satisfactory test exists for the diagnosis of PCP; the various techniques that aim to demonstrate the organism often fail and may be hazardous (Walzer et al., 1974), and serology may, therefore, be preferable. Both complement fixation and immunofluorescence methods have given variable results in different hands (Kagan and Norman, 1976) but we have found that Nowosławski's indirect fluorescent antibody method, using paraffin block sections of infected lung as substrate, is simple and reproducible (Nowosławski and Brzosko, 1964). The antigen responsible is of a polysaccharide nature which is not destroyed by fixation or processing, and the reaction appears to be specific.

It is true that patients with pneumocystis pneumonitis may be concurrently infected with other organisms, for example, cytomegalovirus, and the possibility remains that their sera might contain antibodies which react with other antigens in the lung sections to give positive fluorescence. Our evidence for specificity is that fluorescence is maximal within the alveolar spaces, where methenaminesilver staining of the lung shows that pneumocysts are most abundant. (Other organisms such as viruses are more likely to invade the surrounding cells of the alveolar walls.) Also, the proportion of high or rising titres of antibody in patients with histologically proven PCP provides support for the assumption of specificity. This point will be satisfactorily resolved only when pure in-vitro cultures of pneumocysts can be obtained for use as the substrate as may shortly be possible (Pifer et al., 1977).

Differences in the recorded incidence of positive antibody findings between our results and those of other workers (Norman and Kagan, 1973; Meuwissen et al., 1977) who used dried smears of pneumocysts (made by sucrose-gradient purification and enzyme extraction, respectively) are likely to be related to technique, to the time at which serum samples were taken, and to the titre that is regarded as significant.

Since, in our series, more than half the normal controls with no known contact with PCP were found to have detectable antibody, serological estimations among contacts were shown to have no value in indicating a possible common source of infection. However, this finding did suggest that many apparently healthy people may have had mild or undetectable respiratory infection with $\boldsymbol{P}$. carinii. This is borne out by Meuwissen et al. (1977), who suggest that $100 \%$ of children have been exposed to pneumocystis by the age of 2 years.

As the antibody rise in patients was confined to the IgG class (no rise in IgM was demonstrated), it may be presumed that pneumonitis arose through reactivation of a latent infection caused by immunosuppression resulting from their primary illness and/ or its treatment. Three patients who were proved to have PCP failed to show a significant rise of antibody titre. Two had rather low immunoglobulins (IgG 3.43 and $5.05 \mathrm{~g} / \mathrm{l}$, and $\operatorname{IgM} 0.78$ and $0.46 \mathrm{~g} / \mathrm{l}$, respectively) but one failure (a unique patient with no predisposing cause) was unexplained. With these exceptions, it is apparent that the type of immunosuppression present-mostly drug-induced-did not prevent an antibody response, which was often vigorous. On the other hand, three of the clinically suspected cases failed to show either a fourfold antibody change or a maximum titre above the 'normal' range. A possible explanation is that they 

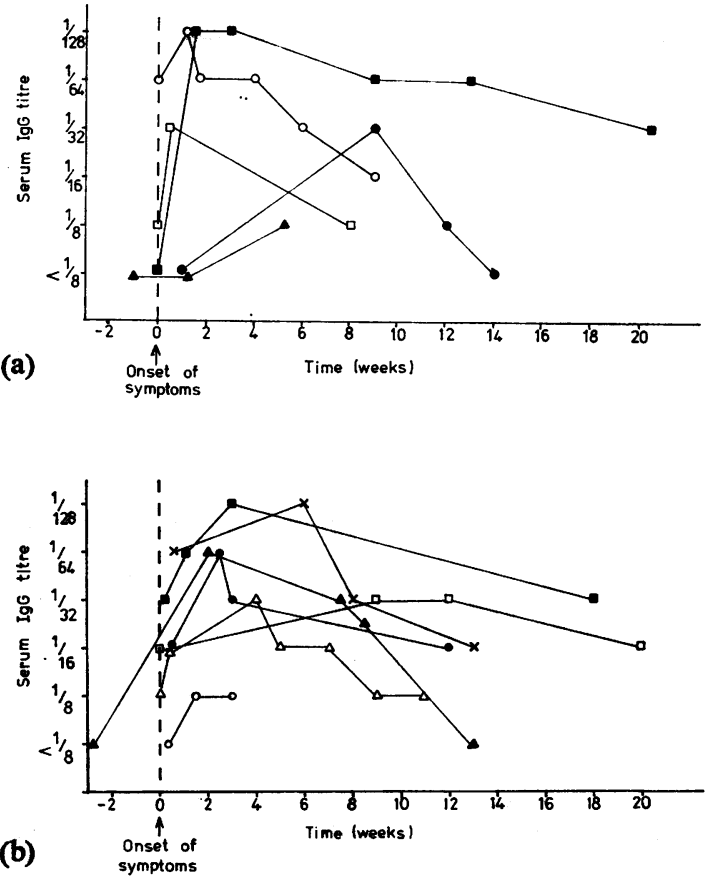

Fig. 2 Serial immunofluorescent IgG titres in PCP: (a) Proven cases

- DD - fourfold rise demonstrated over two months but maximum titre probably missed

O DH - initial 'diagnostic' titre and subsequent fall after one month

CW - rapidly rising titre despite low IgG level; also shows persistence of antibody to 20 weeks at least

$\square$ HP - fourfold rise in three days; no further sample for two months so maximum titre probably missed

$\Delta$ SI - insignificant antibody response (patient also

(b) Suspected cases had low IgG level)

- JK - fourfold rise in two weeks; maximum titre maintained for only five days

$\bigcirc$ GF - insignificant rise, doubtful diagnosis

VM - high titre, rapidly achieved

SR - slight rise and fall demonstrated; higher titre may have occurred during two-month gap

$\Delta \mathrm{KF}$ - rapid rise from negative two weeks pre-onset

$\triangle A N$ - fourfold rise and fall; sample at two weeks might have shown higher titre

$\times$ SL - 'diagnostic' titre at five days followed by further rise and fairly sharp fall

were not infected by $P$. carinii. Two, however, responded to high-dose co-trimoxazole or pentamidine after failing to improve on other antibiotic regimes, but the efficacy of the former is not confined to pneumocystis infections.

The value of serology in PCP is primarily to confirm a diagnosis in retrospect. But since the maximum titre is achieved within a short time of the onset of the infection (some patients already had titres of 1:64 at the time of admission), the finding of an initial antibody level above the arbitrary normal maximum of $1: 32$, or the demonstration of a decisive rise, can be taken as strongly suggestive of PCP. This could be of immediate value in supporting the continuation of specific therapy in the face of side effects such as a co-trimoxazole rash or pentamidine nephrotoxicity.

We express our thanks to Dr Peter Howard for help with the collection of serum samples from the Royal Marsden Hospital patients, and to Dr H. E. M. Kay for advice. We are also grateful to the Bud Flanagan Leukaemia Research Fund for financial support to VS.

\section{References}

Hughes, W. T. (1975). Current status of laboratory diagnosis of Pneumocystis carinii pneumonitis. CRC Critical Reviews in Clinical Laboratory Sciences, 6, 145-170.

Hughes, W. T., Feldman, S., and Sanyal, S. K. (1975). Treatment of Pneumocystis carinii pneumonitis with trimethoprim sulfamethoxazole. Canadian Medical Association Journal, 112, 47S-50S.

Kagan, I. G., and Norman, L. G. (1976). Serology of pneumocystosis. National Cancer Institute Monographs, 43, 121-125.

Lau, W. K., and Young, L. S. (1976). Trimethoprimsulfamethoxazole treatment of Pneumocystis carinii pneumonia in adults. New England Journal of Medicine, 295, 716-718.

Lipson, A., Marshall, W. C., and Hayward, A. R. (1977). Treatment of Pneumocystis carinii pneumonia in children. Archives of Disease in Childhood, 52, 314-319.

Meuwissen, J. H. E. T., Tauber, I., Leewenberg, A. D. E. M., Beckers, P. J. A., and Sieben, M. (1977). Parasitologic and serologic observations of infection with pneumocystis in humans. Journal of Infectious Diseases, 136, 43-49.

Norman, L., and Kagan, I. G. (1973). Some observations on the serology of Pneumocystis carinii infections in the United States. Infection and Immunity, 8, 317-321.

Nowosławski, A., and Brzosko, W. J. (1964). Indirect immunofluorescent test for serodiagnosis of Pneumocystis carinii infection. Bulletin de l'Academie Polonaise des Sciences (Série des Sciences Biologiques), 12, 143147.

Pifer, L. L., Hughes, W. T., and Murphy, M. J., Jr. (1977). Propagation of Pneumocystis carinii in vitro. Pediatric Research, 11, 305-316.

Singer, C., Armstrong, D., Rosen, P. P., and Schotten- 
feld, D. (1975). Pneumocystis carinii pneumonia. A cluster of eleven cases. Annals of Internal Medicine, 82, 772-777.

Walzer, P. D., Perl, D. P., Krogstad, D. J., Rawson, P. G., and Schultz, M. G. (1974). Pneumocystis carinii pneumonia in the United States. Epidemiologic, diagnostic and clinical features. Annals of Internal Medicine, 80, 83-93.

Requests for reprints to: Dr Beryl Jameson, The Bacteriology Department, The Royal Marsden Hospital, Downs Road, Sutton, Surrey, UK. 